\title{
Multi-Fidelity Surrogate-Based Optimization for Electromagnetic Simulation Acceleration
}

\author{
YI WANG and PAUL D. FRANZON, North Carolina State University \\ DAVID SMART and BRIAN SWAHN, Analog Devices Inc.
}

\begin{abstract}
As circuits' speed and frequency increase, fast and accurate capture of the details of the parasitics in metal structures, such as inductors and clock trees, becomes more critical. However, conducting high-fidelity 3D electromagnetic (EM) simulations within the design loop is very time consuming and computationally expensive. To address this issue, we propose a surrogate-based optimization methodology flow, namely multifidelity surrogate-based optimization with candidate search (MFSBO-CS), which integrates the concept of multi-fidelity to reduce the full-wave EM simulation cost in analog/RF simulation-based optimization problems. To do so, a statistical co-kriging model is adapted as the surrogate to model the response surface, and a parallelizable perturbation-based adaptive sampling method is used to find the optima. Within the proposed method, low-fidelity fast RC parasitic extraction tools and high-fidelity full-wave EM solvers are used together to model the target design and then guide the proposed adaptive sample method to achieve the final optimal design parameters. The sampling method in this work not only delivers additional coverage of design space but also helps increase the accuracy of the surrogate model efficiently by updating multiple samples within one iteration. Moreover, a novel modeling technique is developed to further improve the multi-fidelity surrogate model at an acceptable additional computation cost. The effectiveness of the proposed technique is validated by mathematical proofs and numerical test function demonstration. In this article, MFSBO-CS has been applied to two design cases, and the result shows that the proposed methodology offers a cost-efficient solution for analog/RF design problems involving EM simulation. For the two design cases, MFSBO-CS either reaches comparably or outperforms the optimization result from various Bayesian optimization methods with only approximately one- to two-thirds of the computation cost.
\end{abstract}

\section{CCS Concepts: • Hardware $\rightarrow$ Best practices for EDA;}

Additional Key Words and Phrases: Statistical machine learning, surrogate-based optimization, multi-fidelity, electromagnetic simulation

\section{ACM Reference format:}

Yi Wang, Paul D. Franzon, David Smart, and Brian Swahn. 2020. Multi-Fidelity Surrogate-Based Optimization for Electromagnetic Simulation Acceleration. ACM Trans. Des. Autom. Electron. Syst. 25, 5, Article 45 (August 2020), 21 pages.

https://doi.org/10.1145/3398268

This work was funded in part by the NSF under CNS-1624770 and the industry members of the Center for Advanced Electronics through Machine Learning (CAEML) IUCRC.

Authors' addresses: Y. Wang and P. D. Franzon, Monteith Research Center, North Carolina State University, Raleigh, NC 27606; emails: \{ywang73, paulf\}@ncsu.edu; D. Smart and B. Swahn, Analog Devices Inc., 1 Analog Way, Wilmington, MA 01887; emails: \{david.smart, brian.swahn\}@analog.com.

Permission to make digital or hard copies of all or part of this work for personal or classroom use is granted without fee provided that copies are not made or distributed for profit or commercial advantage and that copies bear this notice and the full citation on the first page. Copyrights for components of this work owned by others than ACM must be honored. Abstracting with credit is permitted. To copy otherwise, or republish, to post on servers or to redistribute to lists, requires prior specific permission and/or a fee. Request permissions from permissions@acm.org.

(C) 2020 Association for Computing Machinery.

1084-4309/2020/08-ART45 \$15.00

https://doi.org/10.1145/3398268 


\section{INTRODUCTION}

As the operating frequency of analog/RF circuit blocks increases, detailed electromagnetic (EM) modeling of passive wire structure becomes more difficult and increases time to market. In some high-speed applications, such as flash ADCs, the skew introduced by signal coupling and crosstalk in clock and data distribution paths has a significant effect on performance [23]. Circuits are often added to implement skew calibration and mismatch correction at the expense of added power and circuitry $[20,22]$. Redesigning the physical layout of the interconnect to correct these issues is an alternative that requires expensive iterative EM simulations to ensure rigorous performance. From this perspective, a method to eliminate the high computation cost of multiple full-wave EM simulations would be a priority in the design process. The space mapping (SM) technique [1] was one of the earliest approaches used to reduce this cost. With this technique, coarse and fine EM simulation techniques are combined to reduce overall simulation time. In this technique, a linear mapping is found between coarse and fine model input parameter spaces by using an iterative process to evaluate the error between fine response surface (RS) and coarse RS. Later, variants of SM merged to enhance the mapping function $[10,11,13]$. However, SM techniques still require sufficiently faithful coarse models to assure good results, and more sample data may not lead to improvement in the model accuracy [12]. Beyond that, most work related to SM techniques focuses on the microwave device modeling rather than a systematic optimization methodology to acquire optimal design parameters, such as for circuit design. Recently, Bayesian optimization (BO) [24], a surrogate-based optimization technique, has been shown to be useful in the investigation of simulation-based analog/RF ICs design automation where a large scale of expensive SPICE simulations have to be executed to obtain the design performance [15, 21, 30]. Compared with the traditional direct optimization methods like gradient-based or evolutionary-based ones [28], BO requires much fewer samples to find the proper optima. However, it is still worth investigating other algorithms to further reduce the total computation cost and be capable to handle high-dimensional design problems.

This proposed work emphasizes a cost-effective solution toward the EM simulation-based optimization in analog/RF design problems. Rather than simple utilization of a costly fine EM evaluation dataset, we employ EM simulation data at two different levels of sophistication: a large number of low-fidelity simulations and a small number of high-fidelity simulations. These are fitted to a stochastic model for predicting the uncovered area in the design space. The concept of multi-fidelity model-ing is frequently implemented in the area of aerospace engineering since it is common to take over one day for producing a single low-resolution plot using physical simulators [4-7]. The utilization of low-fidelity emulation data could assist the system to quickly capture the approximate high-fidelity RS by evaluating large amount of low-fidelity samples at relatively low computation cost. In EM simulation, we define the high-fidelity simulators as 3D or 2.5D EM solvers whose algorithms rely on the method of moments (MoM), finite element method (FEM), or finite difference time domain (FDTD) [27]. Even within each specific high-fidelity simulator, various settings, such as edge mesh, thickness, the 3D model, and ration, could lead to different levels of fidelity at the trade-off between simulation accuracy and computation cost. This provides a wider selections of multi-fidelity simulators for EM simulation acceleration. In this work, the low-fidelity simulators adopted are ASITIC [18] and a commercial parasitics extraction tool for the two separate design cases. ASITIC relies on the simple pi model to derive S-parameters to a circuit representation. The basic algorithm behind the commercial parasitics extraction tool is to calculate the parasitics by geometrically analyzing conductors in 3D regions.

Apart from employing a multi-fidelity model as the surrogate, our proposed method leverages the cheap evaluation cost of the surrogate model by conducting a large number of surrogate 
evaluations. The current optima and its corresponding design inputs are obtained first. Then the surrogate evaluations are executed by varying the inputs near the current optima and within the entire design space. Contrary to traditional BOs, there is no extra effort in balancing current surrogate model exploitation and uncovered design space exploration through optimization of acquisition function. The model exploration is simply fulfilled with the perturbation of input variables in the whole design space due to the low evaluation cost of the surrogate model. Specifically, in the sequential sample method applied, we generate multiple groups of samples that have different perturbation properties in a parallel, and the variables to be perturbed are selected following a dropout style [8]. The surrogate determines the best sample point so far in each group and then runs the expensive EM simulation on these points. Once the improvement from an expensive 3D EM solver (high-fidelity) simulation is detected, not only would the high-fidelity data be used to update the surrogate but also the system would re-evaluate this improved point in the low-fidelity simulator and update the low-fidelity dataset to further acquire a higher accuracy and resolution in this area of interest. The main contributions of the work are as follows:

- To the best of our knowledge, this is the first work adapting the concept of multi-fidelity (co-kriging) with the proposed adaptive sampling method to EM simulation-based optimization.

- A sampling method is employed that generates multiple disparate samples at each step of the algorithm, including perturbated samples, the next sample in the Sobol sequence, and a random sample. This approach helps the algorithm avoid local minima.

- The adaptive sampling method developed in this work not only provides a better design space coverage but also reduces the elapsed time required for the optimization since several sample points are evaluated in parallel. As the the concept of dropout is adopted, the proposed method will be a fit for high-dimensional design problems.

- The mechanism of appending the low-fidelity samples to update the surrogate during optimization yields a simple way to increase the multi-fidelity model accuracy at certain areas where the potential global optimum lies.

- The proposed methodology is proved to be more efficient in both simulation time and computation cost compared with the state-of-the-art BO for the examples presented in this work.

The rest of the article is organized as follows. In Section 2, we present the problem formulation and an overview of the proposed multi-fidelity surrogate-based optimization with candidate search (MFSBO-CS) for the EM simulation acceleration problem. The detailed features of each block are described in Section 3, and the key contributions of the work are reported in Sections 3.3, 3.5, and 3.6. Section 4 reviews the BO methods as the baseline for comparison with this work. Section 5 demonstrates the numerical results of two design cases along with the comparison between the proposed work and BO. Section 6 concludes the article.

\section{OVERVIEW OF EM SIMULATION ACCELERATION}

\subsection{Problem Formulation}

The generic definition of an engineering inverse problem is to find a set of input design variables that can achieve given output performance goals. In this work, solving the inverse problem will be addressed in the context of optimization. Therefore, the methodology developed is to minimize the difference between the objective function value and a target value, where the objective function values are acquired from multi-fidelity EM simulations. Let $f(x)$ denote the objective function, given the input $\mathbf{x}$ and target value $T$. The solution to inverse problem $x^{*}$ can be rewritten in the 


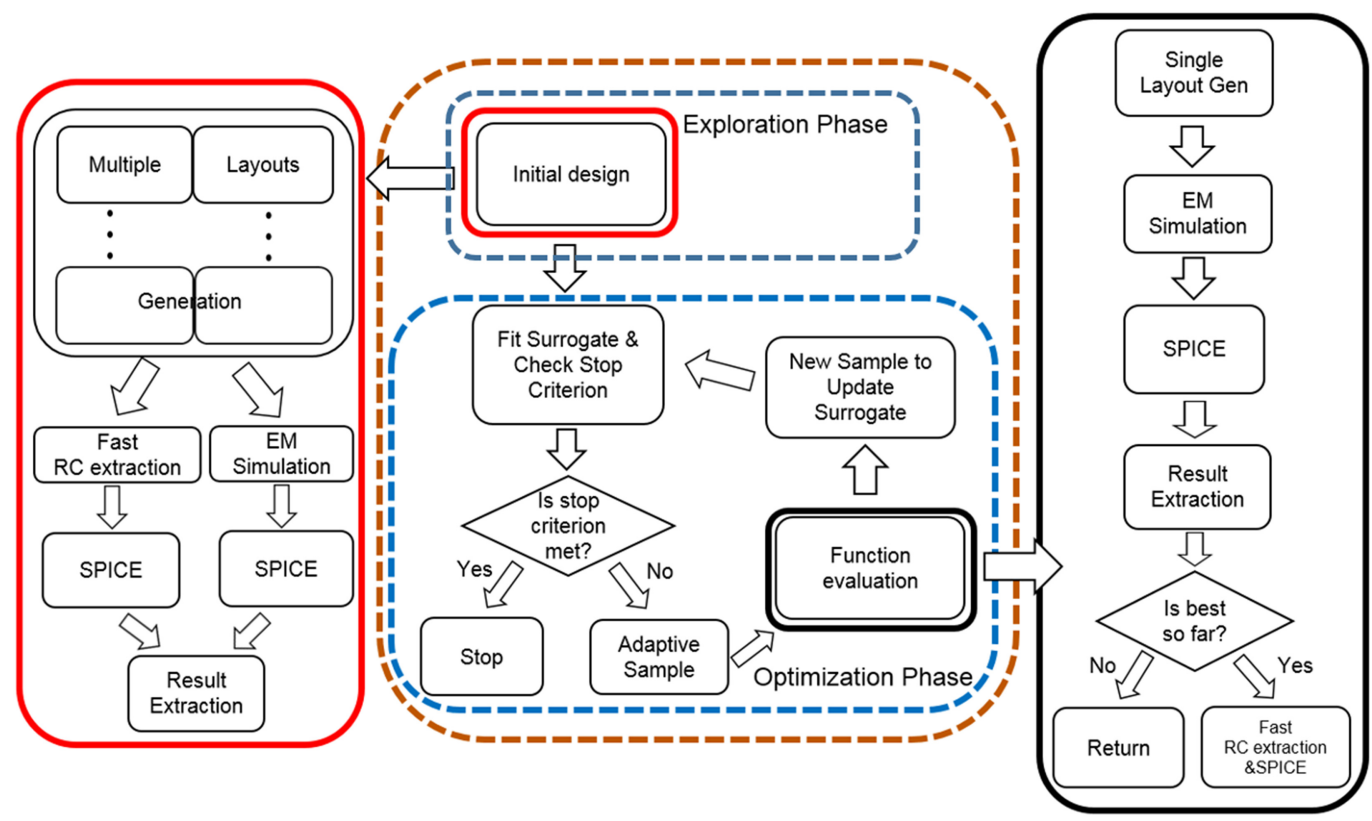

Fig. 1. Overview of MFSBO-CS.

form of solving an optimization problem as follows:

$$
x^{*}=\arg \min _{x \in R}\|f(x)-T\| .
$$

Our task is to approach the $x^{*}$ that satisfies $\|f(x)-T\|=0$ in an efficient way by avoiding extensive costly EM simulations and thus accelerate the analog/RF design process.

\subsection{Overview of MFSBO-CS}

In this section, we decompose the whole framework of the proposed method into several pieces. The complete overview of the proposed MFSBO-CS for clock tree optimization is illustrated in Figure 1. There is slight difference in representing the flow chart for the inductor design case considering that inductor design only involves multi-fidelity EM simulations. From a high-level perspective, the proposed approach consists of four major components: (1) design exploration, (2) surrogate model construction, (3) adaptive sampling, and (4) model evaluation. The core of our approach is to utilize a statistical surrogate model trained from multi-fidelity data to search for the global optima with the fewest samples. A surrogate model is a model fitted to evaluations of a detailed model (that often takes a long time to evaluate). It thus serves as a "surrogate" for the detailed model. It is sometimes called a response surface (RS). Conventionally, a surrogate model is fitted to evaluations of only one detailed or fine model. In this work, it is fitted to a combination of fine model evaluations and (faster) coarse model evaluations-hence the term multi-fidelity.

\section{MFSBO-CS FLOW}

Algorithm 1 shows the detailed MFSBO-CS flow that we developed. The flow is initialized with a multi-fidelity dataset obtained from a "design of experiment" (DoE; lines 2 through 14). Then we build a multi-fidelity statistical model based on the dataset acquired from the previous step (line 15). This surrogate model is updated iteratively as coded from step 16 to step 38 . The iteration will end when the total simulation budget $T$ runs out. 


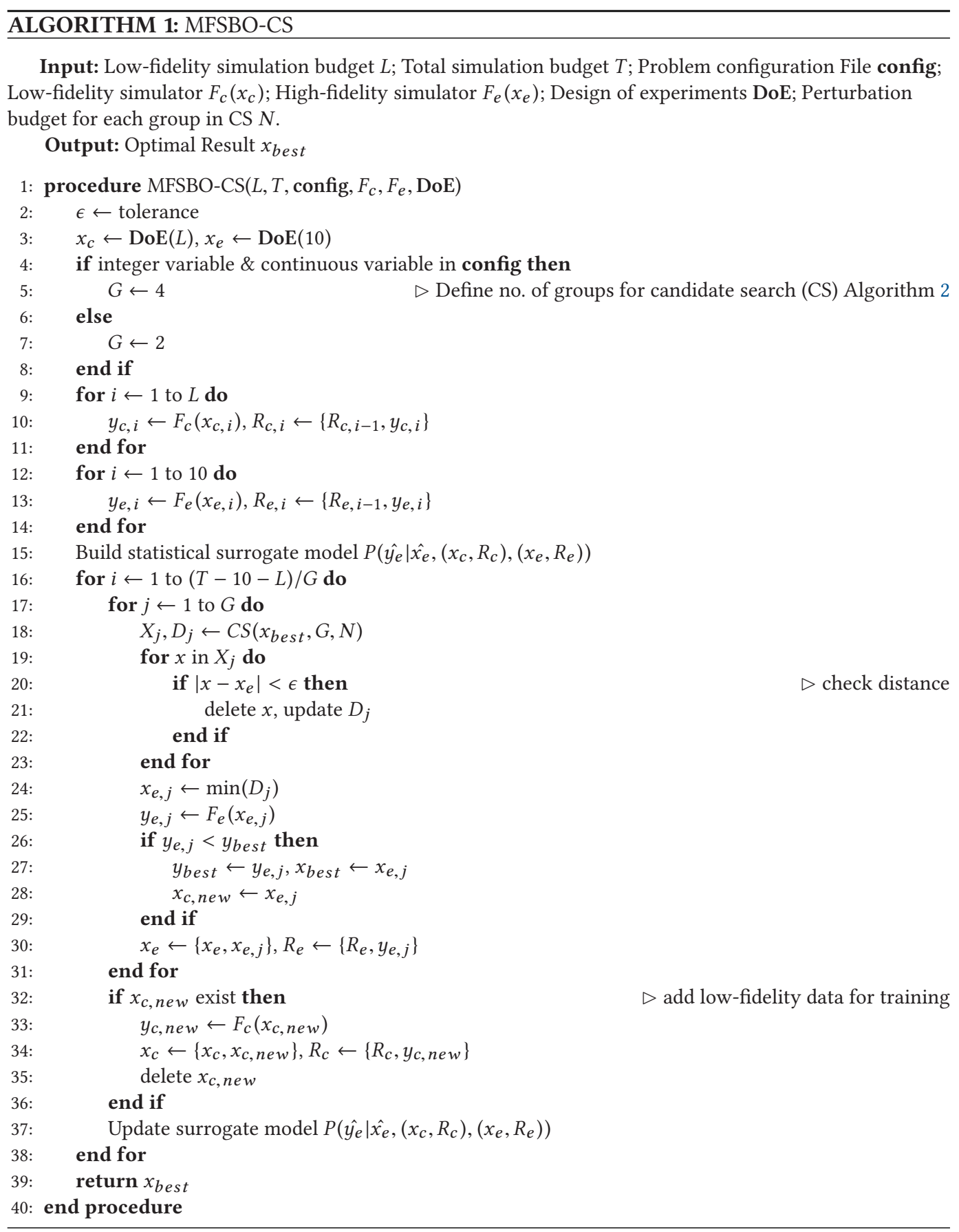

\subsection{Design Exploration}

In traditional surrogate-based optimization, the aim of the design exploration stage is to build an initial RS for the next optimization stage by evaluating a number of designed points with only highfidelity simulations. Generally, a layout structure EM optimization problem RS is highly non-linear 
Table 1. Low- and High-Fidelity Average Simulation Cost (in seconds)

\begin{tabular}{ccl}
\hline Simulator & Simulation Time Cost & \multicolumn{1}{c}{ Comment } \\
\hline ASITIC & 0.125 & For inductor case \\
EM Solver 1 & 76.583 & For inductor case \\
RC extraction simulator & $386 e^{-0.64 n}+55.16$ & For clock tree case \\
EM Solver 2 & 138.012 & For clock tree case \\
\hline
\end{tabular}

Note: The EM solvers are the high-fidelity solvers, and the others are low-fidelity ones.

and non-convex, so we do not have much prior information about how to sample this black box. The most straightforward way to find the approximate optima is to sample the design space with uniform coverage and then evaluate the samples using expensive simulations. Among the existing sampling methods, the Latin hypercube sampling (LHS) [16] is a simple and effective choice. This method ensures uniformly spread projections of all points on all axes. Even if an effective initial sampling method is used, it is obvious that the more data we have, the more accurate the surrogate model will be. Therefore, it encapsulates the dilemma that training a good surrogate requires more costly EM simulations. However, in this work, this situation is alleviated by the surrogate model construction using both cheap RC extraction simulations and expensive EM simulations.

For the multi-fidelity surrogate-based optimization framework developed in this work, we adopted LHS [26] as the design exploration method for both high- and low-fidelity simulations (line 3 of Algorithm 1). Design exploration is also known as design of experiments (DoE) in some literature and is so named in line 3.

In Table 1, we also summarize the average simulation cost for two sets of multi-fidelity simulators used in this article. The EM solvers are the high-fidelity solvers, whereas ASITIC and RC extraction simulator are low-fidelity ones. Between lines 9 and 13 of Algorithm 1, the samples generated from DoE will be evaluated with low-fidelity simulator $F_{c}\left(x_{c}\right)$ at $x_{c}$ and high-fidelity simulator $F_{e}\left(x_{e}\right)$ at $x_{e}$.

In Algorithm 1, the initial surrogate is built with $L$ evaluations of the low-fidelity model at $x_{c}$ and 10 evaluations of the high-fidelity model at $x_{e}$. The number of low-fidelity model evaluations $L$ should be larger than $2 *(D+1)$, where $D$ is the dimension of the design. The requirement for the number of high-fidelity evaluations needs to be larger than $D$ since the inverse of the covariance matrix could not be calculated if the number of high-fidelity samples is smaller than $D$.

For the inductor layout design example, the low-fidelity simulator is approximately $620 \mathrm{x}$ faster than the high-fidelity EM solver, thus making the multi-fidelity model building very effective.

In the clock tree case, we developed a novel working flow to execute fast RC parasitic extraction (low-fidelity simulation) as follows. First, generate multiple clock tree layouts in different sizes based on the $x_{c}$. Second, merge all single layouts into one. Third, run RC extraction and SPICE simulation for the single combined layout. Fourth, analyze and synthesize the timing result from simulation. The procedure is shown on the left side of initial design block in Figure 1. Based upon the description of running low-fidelity simulations, it is easy to discover that the simulation time of $n$ layouts does not simply follow $\mathrm{O}(n)$. As the number of the synchronous layout RC extraction simulation increases, the average computation cost for completing one simulation decreases because of the mechanism used in the flow. Thus, the sampling cost is faster than $\mathrm{O}(n)$. As a matter of fact, we used a logarithmic regression model to fit the simulation time data, and the result is shown in Table 1. It indicates that this set of multi-fidelity simulators will be a good candidate for the framework.

We follow the assumption, also used in the work of Kennedy and O'Hagan [9], that the high-fidelity simulators are trustworthy and all errors occur in the low-fidelity simulators. A 
multi-fidelity model is constructed based upon the samples obtained from design exploration stage as indicated in line 15 of Algorithm 1. The optimum of the high-fidelity samples from the exploration phase will be selected as the starting point of the following optimization phase. The number of high-fidelity simulation samples in the design exploration stage is required to be larger than the problem dimension since we utilize the Gaussian process as our surrogate. The inversion of the covariance matrix cannot be calculated when the dimension is larger than the number of samples.

Now that we have proper cheap and expensive simulators set up to evaluate EM effects in our layout design, we introduce the details of the stochastic multi-fidelity model to bridge the lowfidelity and high-fidelity data in the next section.

\subsection{Statistical Surrogate Model}

Co-kriging [9], a statistical method to construct the mapping function between input and output from data in multiple levels of fidelity, is used in this work. An $n$-sample low-fidelity dataset and its corresponding function evaluation result set are defined as $x_{c}$ and $R_{c}$, respectively. Similarly, $x_{e}$ denotes an $m$-sample high-fidelity dataset, and $R_{e}$ represents its corresponding function evaluation result set. Then the high-fidelity simulator could be expressed in a form of a scaled Gaussian process that denotes the low-fidelity simulator plus another Gaussian process that represents the difference between a high-fidelity simulator and a low-fidelity simulator:

$$
Z_{e}=\rho Z_{c}+Z_{d}
$$

where $Z_{e}$ is the resulting model, $Z_{c}$ is the model for the low-fidelity points only, $Z_{d}$ is the model describing the difference between high-fidelity points and low-fidelity points, and $\rho$ is the correction factor.

We can approximate the covariance matrix of the high-fidelity Gaussian process to be [4]:

$$
V=\left(\begin{array}{cc}
\sigma_{c}^{2} \Phi_{c}\left(x_{c}, x_{c}\right) & \rho \sigma_{c}^{2} \Phi_{c}\left(x_{c}, x_{e}\right) \\
\rho \sigma_{c}^{2} \Phi_{c}\left(x_{e}, x_{c}\right) & \rho^{2} \sigma_{c}^{2} \Phi_{c}\left(x_{e}, x_{e}\right)+\sigma_{d}^{2} \Phi_{d}\left(x_{e}, x_{e}\right)
\end{array}\right),
$$

where $\Phi_{c}$ and $\Phi_{d}$ denote the covariance matrix of $Z_{c}$ and $Z_{d}$, respectively. To fit the hyperparameters $\theta_{c}, \theta_{d}$ (unknown scaling factors in covariance function), $\mu_{c}, \mu_{d}$, and $\rho$, we perform maximizing likelihood estimate (MLE) on both $Z_{c}$ and $Z_{d}$.

Thus, the final posterior mean of $Z_{e}$ given a new sample $\mathrm{x}$ is

$$
\mu_{e}(x)=q(x)^{T} \alpha+k(x)^{T} V^{-1}(y-B \alpha)
$$

where

$$
\begin{gathered}
q(x)=\left(\rho b(x)^{T}, b(x)^{T}\right), \\
\alpha=\left(B^{T} V^{-1} B\right)^{-1} B^{T} V^{-1} y, \\
k(x)^{T}=\left(\rho \sigma_{c}^{2} \Phi_{c}\left(x, x_{c}\right), \rho^{2} \sigma_{c}^{2} \Phi_{c}\left(x, x_{e}\right)+\sigma_{d}^{2} \Phi_{d}\left(x, x_{e}\right)\right), \\
B=\left(\begin{array}{cc}
b\left(x_{1}^{c}\right)^{T} & 0 \\
\cdots & \cdots \\
b\left(x_{n}^{c}\right)^{T} & 0 \\
\rho b\left(x_{1}^{e}\right)^{T} & b\left(x_{1}^{e}\right)^{T} \\
\ldots & \cdots \\
\rho b\left(x_{m}^{e}\right)^{T} & b\left(x_{m}^{e}\right)^{T}
\end{array}\right) .
\end{gathered}
$$

The preceding $b(x)$ is a vector of polynomial regression functions fitted during the training process that describes $Z_{c}$ roughly over the entire design space. In the ordinary kriging, the model is in a 


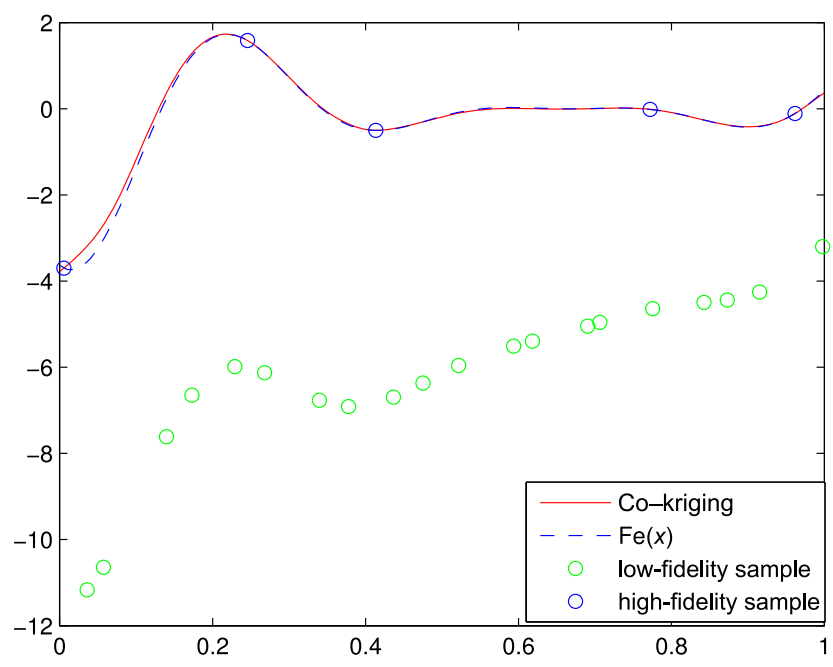

Fig. 2. Co-kriging model performance.

format of a regression function $f(x)$ plus a Gaussian process $Z$. The regression $f(x)$ is constructed to describe the rough trend (largest variance) of the RS and a Gaussian process $Z$ is built to gap the residuals. In this case, $f(x)$ is modeled as a weighted sum of polynomials $\sum a_{i} b_{i}(x) . \Phi_{c}\left(x, x_{c}\right)$ represents the correlation vector between the point to be predicted, namely $x$, and the sample data points in low-fidelity $x_{c}$. It is a vector whose entries are calculated by the low-fidelity covariance function. $\Phi_{c}\left(x, x_{e}\right)$ denotes the correlation vector between the point to be predicted and the sample data points in high fidelity. The low-fidelity covariance function is applied as well to obtain the entries in the vector.

To visualize the modeling performance of co-kriging, a 1D example is used as an illustration. Assume the high-fidelity function and the low-fidelity function as

$$
\begin{gathered}
F_{e}(x)=(3 x-2)^{2} \sin (15 x-2), x \in(0,1), \\
F_{c}(x)=0.8(3 x-2)^{2} \sin (15 x-2)+5(x-0.5)-6, x \in(0,1) .
\end{gathered}
$$

We execute the flow from line 3 to line 15 with 20 low-fidelity and 5 high-fidelity samples. The green dots depicted in Figure 2 are 20 LHS sampled points in range of $(0,1)$ with values obtained from the low-fidelity simulator equation (10). Similarly, a high-fidelity dataset of 5 points are generated and depicted as the blue dots in the figure. The co-Kriging model is built based on these 25 samples using the mean estimator shown in Equation (4). The red line in the figure is plotted by sampling the trained co-kriging model in the interval of 0.01 . The blue dashed lines are generated by sampling $F_{e}(x)$ at the step of 0.01 .

The co-kriging model performance shown in Figure 2 has a leave-one-out cross-validation error of 0.011999 and integrated mean square error of 0.005919 . Thus, co-kriging predictions aligns with the exact high-fidelity function curve very well for this $1 \mathrm{D}$ problem. Co-kriging provides an excellent choice of statistical surrogate that can be built with a combination of the data from high-fidelity EM simulation and the low-fidelity RC extraction tool.

\subsection{Adaptive Sampling with Dropout}

One-shot samples from the design exploration are usually not enough to construct the globally feasible model due to many unknown non-linearity and discontinuities in the target mapping 
function. An adaptive sampling strategy allows a search for new samples, whose locations are not explored yet, by analyzing the current RS. The adaptive sampling method developed in this work is adapted and improved from the work of Müller et al. [17]. The sampling method was improved by adding Sobol and random samples and introducing additional hyperparameters. The number of groups to be generated, $G$, was decided in Algorithm 1. At each iteration, $N$ points are sampled in each of sets X1 to X3 (if $G=4$ ) or set X1 (if $G=2$ ). Nadditional points are sampled using a Sobol sequel or randomly (sets X4 or X2). ( $N$ is the perturbation budget for each group-see Algorithm 1 ). The split between the Sobol set or random set are decided by the hyperparameter $w$, which leads to better coverage of the design space. In addition, the execution of the candidate group generation runs in parallel to further reduce the simulation time cost.

3.3.1 Mixed Integer Optimization. Unlike BO, the adaptive sampling method in this article does not use an acquisition function. When dealing with optimization tasks that involve continuous and discrete variables, a common solution used in BO is to apply binning, or replace the continuous acquisition function optimization value with the closest integer. This can lead to a mismatch between the points where the acquisition takes high values, and where the actual evaluation is performed. This issue can be avoided by finding the optimal next sample point with the adaptive sample strategy proposed in this work. Rather than running an optimization over a function with the posterior mean and covariance estimation information, the samples are generated using a perturbation and evaluated with the existing surrogate model directly. The next best point will be selected based on the posterior mean estimation, which skips the binning operation and makes it fit for mixed integer optimization problems [3]. Algorithm 2 illustrates the details of proposed sampling method that is embedded in Algorithm 1 at line 18.

3.3.2 Dropout for High-Dimension Problems. A key feature of Algorithm 2 is the application of the concept of dropout [14], which is a widely used technique for training deep neural nets [29]. In that context, the concept is used to probabilistically remove (or "drop out") neurons in the network. Here, it is used to remove dimensions, so as to reduce the modeling cost. The variables to be perturbated will be randomly selected when the number of the dimensions in the design space is high (i.e., the dimensions not selected are dropped), which is similar to the random dropout of a certain percentage of neurons in one layer. If both discrete and continuous variables are detected in the problem configuration file, an internal variable, group size $G$ would be set to 4; otherwise, if only continuous variables are detected, $G$ equals to 2 (Algorithm 1, lines 4 through 8). The inputs of the proposed candidate search (CS) sampling method are the current optimal sample $x_{\text {best }}$, group size $G$, and perturbation budget $N$ for each group (Algorithm 1, line 18). The group size $G$ denotes how many groups of sequential samples with different location properties are generated in parallel. Perturbation budget $N$ for each group means that $N$ samples will be generated for each group. For the $G=4$ case, we will have a group (G1) of $N$ samples only performing perturbation on continuous variables of the current best point by randomly selecting continuous variables from all continuous variables. This generates the set X1 of new points to sample. Only "indexint" of the "indent" dimensions are sampled, and that set is randomly selected (i.e., the unsample dimensions are dropped). The second group (G2) has $N$ samples based only on perturbations on random chosen discrete variables of the current best point. This generates the set X2. The third group (G3) is formed by perturbing the randomly selected continuous and discrete variables of the current best point. This generates the set X3. The fourth group (G4) contains the $N$ points sampled from the whole design space, generating set X4. The perturbation rates for groups G1 through G3 are stored in a table for each chosen variable. The perturbation rate for each chosen variable is randomly selected in that table. The essence of the sampling method makes it a good candidate for highdimensional problem optimization since not all design variables are used in the process. 


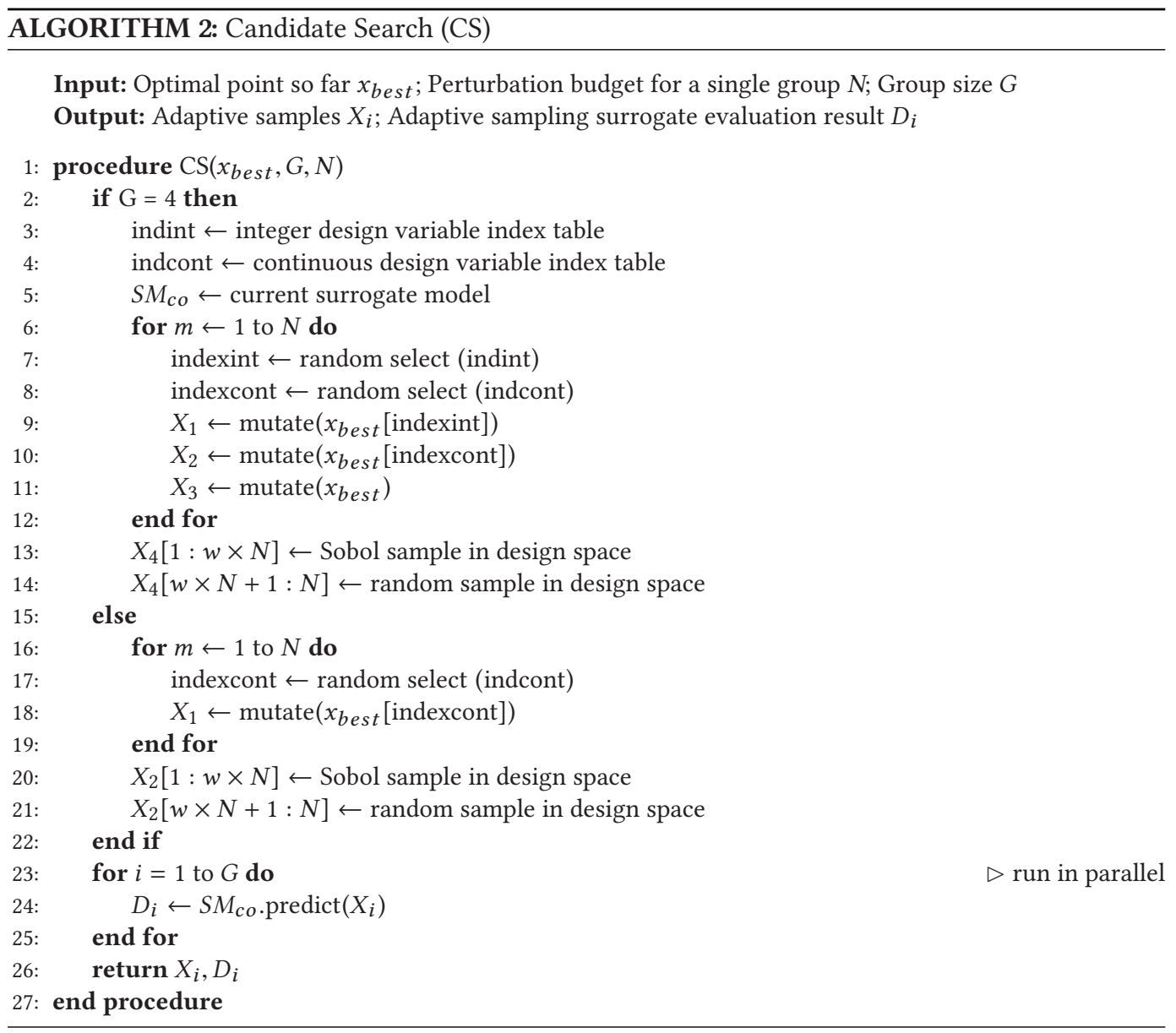

Specifically, when it comes to generating the fourth group of samples, part of the perturbation budget will assign to generate random samples, and the rest is designed to be low-discrepancy sequence samples to ensure sufficient coverage of the design space. The low-discrepancy sample sequence adopted in this work is Sobol sequence sampling [25]. The Sobol sequence was chosen because it produces very uniformly distributed samples. The weight $w$ between the random sampling count and Sobol sampling count was adjustable, and 0.2 was found to be suitable in the experiments described in this article (Algorithm 2, lines 20 and 21). All of these points X1, X2, X3, and $\mathrm{X} 4$ are evaluated with the current surrogate model SM (Algorithm 2, line 24). These points and evaluations then become the set of points, $X_{i}$, and the set of results, $D_{i}$, referred to in line 18 of Algorithm 1.

\subsection{Adaptive Samples Filtering}

After completing the adaptive sampling method CS, a distance checking step is performed as described in lines 19 through 22 in Algorithm 1 . The distance between the newly generated samples and existing samples that have already been stored in $x_{e}$ is calculated using $k$-nearest neighbors $(\mathrm{KNN})$. If the distance of a newly sampled point to the nearest existing training sample is shorter than the preset $\epsilon$, it will be discarded. If there was no such distance checking, it is highly likely 
that the potential minimum of $D_{i}$ would aggregate at the location where the current known optimum lies (i.e., get stuck at a local optimum). As a result, the optimization result could not be further improved and converges to a bad value. As the stopping criteria is not met, the system would keep computing those repetitive samples with a costly high-fidelity simulator, which leads to much waste in computation.

\subsection{Low-Fidelity Dataset Update}

A new technique is introduced to improve surrogate model accuracy at acceptable additional cost as described in lines 24 through 36 in Algorithm 1.

3.5.1 Explanation and Mathematical Proof with Some Approximation. The next step after filtering the samples is to find the sample with the best surrogate model value from each group and evaluate them with the high-fidelity EM simulator as shown in lines 24 and 25 in Algorithm 1. Once a sample whose value is better than the existing optima is found, we will record and resimulate it with the low-fidelity simulator. After that, it will be appended to the low-fidelity dataset for the surrogate model update. This process is coded from lines 26 to 36 in Algorithm 1. This technique probably results in a better surrogate modeling accuracy at those certain areas where the potential optimum lies, since the samples added back to the low-fidelity dataset are temporarily optimal in high-fidelity space. The only extra cost of implementing this action is to execute one additional low-fidelity simulation when a verified high-fidelity optimum is detected.

We will derive the math intuitively for the procedure described earlier to prove its feasibility in model accuracy improvement. Suppose that a new low-fidelity sample $x^{*}$ is added back to the multi-fidelity training dataset; the covariance matrix $V$ could be expressed in the extended format of (3) as follows:

$$
V=\left(\begin{array}{ccc}
\sigma_{c}^{2} \Phi_{c}\left(x_{c}, x_{c}\right) & \rho \sigma_{c}^{2} \Phi_{c}\left(x_{c}, x_{e}\right) & \rho \sigma_{c}^{2} \phi_{c}\left(x_{c}, x^{*}\right) \\
\rho \sigma_{c}^{2} \Phi_{c}\left(x_{e}, x_{c}\right) & \rho^{2} \sigma_{c}^{2} \Phi_{c}\left(x_{e}, x_{e}\right)+\sigma_{d}^{2} \Phi_{d} \Phi_{d}\left(x_{e}, x_{e}\right) & \rho \sigma_{c}^{2} \phi_{c}\left(x_{e}, x^{*}\right) \\
\rho \sigma_{c}^{2} \phi_{c}\left(x^{*}, x_{c}\right) & \rho \sigma_{c}^{2} \phi_{c}\left(x^{*}, x_{e}\right) & \sigma_{c}^{2}
\end{array}\right) .
$$

For calculation purpose, $V$ is partitioned as

$$
V=\left(\begin{array}{cc}
M & P \\
P^{T} & \sigma_{c}^{2}
\end{array}\right),
$$

where

$$
\begin{gathered}
M=\left(\begin{array}{cc}
\sigma_{c}^{2} \Phi_{c}\left(x_{c}, x_{c}\right) & \rho \sigma_{c}^{2} \Phi_{c}\left(x_{c}, x_{e}\right) \\
\rho \sigma_{c}^{2} \Phi_{c}\left(x_{e}, x_{c}\right) & \rho^{2} \sigma_{c}^{2} \Phi_{c}\left(x_{e}, x_{e}\right)+\sigma_{d}^{2} \Phi_{d}\left(x_{e}, x_{e}\right)
\end{array}\right), \\
P=\left(\begin{array}{c}
\rho \sigma_{c}^{2} \phi_{c}\left(x_{c}, x^{*}\right) \\
\rho \sigma_{c}^{2} \phi_{c}\left(x_{e}, x^{*}\right)
\end{array}\right) .
\end{gathered}
$$

From the inspiration of log likelihood function in MLE to determine the hyperparameters, the posterior mean of the newly updated co-kriging could be estimated as maximizing the following multiplied matrix:

$$
-\frac{1}{2}\left(\mathbf{y}-\mu y\left(x^{*}\right)-\mu\right) V^{-1}\left(\begin{array}{c}
\mathbf{y}-\mu \\
y\left(x^{*}\right)-\mu
\end{array}\right) .
$$

After calculating partitioned matrix inverse and partial derivative over $y\left(x^{*}\right)$, we obtain

$$
y\left(x^{*}\right)=\mu+P^{T} M^{-1}(\mathbf{y}-\mu)
$$


where $\mu$ could be estimated by doing partial derivative over $\mu$ in (15). Then the mean square error could be approximated as

$$
e \approx \sigma_{c}^{2}-P^{T} M^{-1} P
$$

Given the mean square error of the co-kriging predictor presented in [4],

$$
e \approx \rho^{2} \sigma_{c}^{2}+\sigma_{d}^{2}-c^{T} C^{-1} c
$$

where $C=M$,

$$
c=\left(\begin{array}{c}
\rho \sigma_{c}^{2} \phi_{c}\left(x_{c}, x^{*}\right) \\
\rho^{2} \sigma_{c}^{2} \Phi_{c}\left(x_{e}, x^{*}\right)+\sigma_{d}^{2} \Phi_{d}\left(x_{e}, x^{*}\right)
\end{array}\right) .
$$

It is easy to discover that the only difference between $P^{T} M^{-1} P$ and $c^{T} C^{-1} c$ is the term of $m \times 1$ dimension matrix $\sigma_{d}^{2} \Phi_{d}\left(x_{e}, x^{*}\right)$ in $c$ supposing that the number of high-fidelity samples and lowfidelity samples are $m$ and $n$. For multi-fidelity optimization, there is no doubt that we would use a larger amount of low-fidelity data than the high-fidelity one. Thus, $n \gg m$ and only a small number of term $\sigma_{d}^{2} \Phi_{d}\left(x_{e}, x^{*}\right)$ are eliminated when the case of appending a low-fidelity sample happens. As a consequence, we obtain

$$
P^{T} M^{-1} P \approx c^{T} C^{-1} c
$$

Then the difference in error (Equations (17) and (18)) can be expressed as

$$
\rho^{2} \sigma_{c}^{2}+\sigma_{d}^{2}-\sigma_{c}^{2} \approx\left(\rho^{2}-1\right) \sigma_{c}^{2}+\sigma_{d}^{2} .
$$

For the design cases we have conducted, the scaling factor $\rho$ varies from 0.9 to 2.3. Intuitively, (21) would be larger than zero in most scenarios, and we could partially prove that the mean square error could be alleviated by additional low-fidelity samples.

3.5.2 Numerical Test on the Developed Technique. There is still some uncertainty about the derivation presented earlier due to the unknown $\rho, \sigma_{c}^{2}$, and $\sigma_{d}^{2}$ that need to be calculated from MLE. Therefore, a numerical experiment was performed to decipher the math of the proposed method using the multi-fidelity test functions (9) and (10). Two performance metrics-mean square error and leave-one-out cross-validation-are evaluated to test the model accuracy.

We first built a co-kriging model with 20 low-fidelity and 5 high-fidelity samples-that is, the point where the number of recursive low-fidelity samples equals to 0 as depicted in Figure 3. In the next step, we sequentially add one high-fidelity sample input at a time to the low-fidelity dataset-that is, at $x=1$, model performance is calculated based on 20 low-fidelity samples, 5 high-fidelity samples, and 1 new added low-fidelity sample. The input variables of this new added low-fidelity sample come from one of the high-fidelity samples. It is then evaluated with the lowfidelity simulator. At $x=2$, there are two randomly selected high-fidelity sample inputs from the 5 high-fidelity sample dataset being added to the low-fidelity dataset after low-fidelity evaluations. After low-fidelity function evaluations, the co-kriging model is retrained to fit the new dataset. This process will be repeated five times until all 5 high-fidelity sample inputs are inserted to the low-fidelity dataset. Figure 3 illustrates the model performance where $x$ denotes the number of the samples added to low-fidelity dataset and $y$ denotes the model performance metrics. The model accuracy is improved by adding more low-fidelity data that coincides with high-fidelity samples and ultimately gets saturated since no more high-fidelity information could be extracted from the training dataset. 

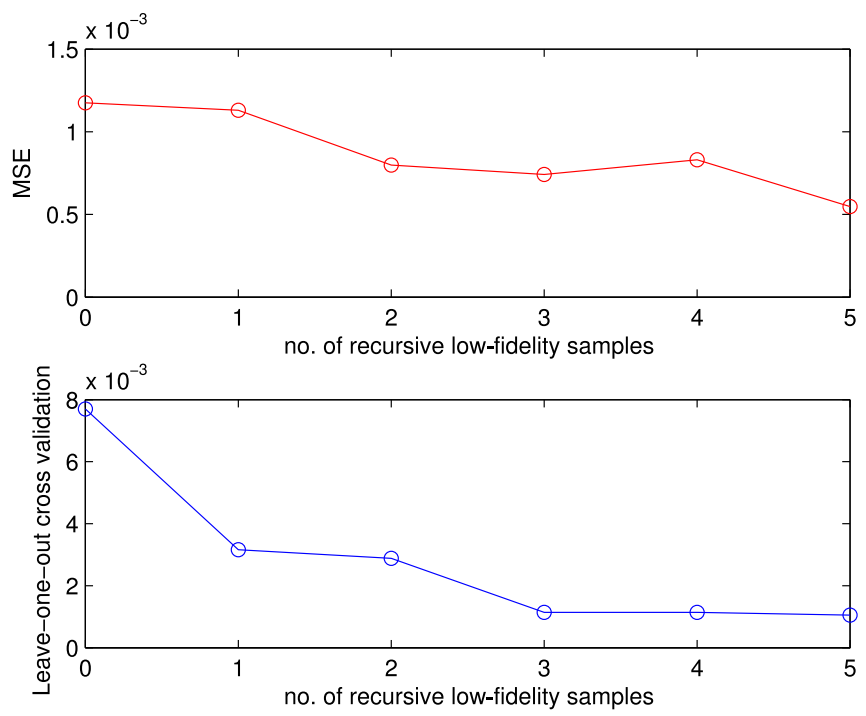

Fig. 3. Model performance improvement vs. number of recursive LF samples.

\subsection{Sample Generation for Model Rebuild}

As depicted at line 23 in Algorithm 2, the surrogate would be updated with G samples in every iteration until the stop criteria is met. The mechanism that generates multiple high-fidelity samples simultaneously to update the surrogate not only accelerates modeling convergence but also naturally leads to a balance in exploring the local area near the current optimum versus the entire design space. As long as the best point in G4 does not violate the distance checking requirement, at least one point generated globally would be added into the training dataset to update the surrogate model.

\section{BASELINE: BO FRAMEWORK}

\subsection{Overview of $\mathrm{BO}$}

$\mathrm{BO}$, another popular approach to optimize expensive black box function was evaluated and implemented for the two design cases in this work as a baseline for benchmarking. Standard BO consists of two components: a Gaussian process as the surrogate to model the black box function, and an acquisition function as the sampling method to decide where to sample next to approach the optimum $[15,19,21,30]$. It is called an acquisition function, as a function is formulated to help decide which point will be most useful to simulate in the next step of the optimization procedure (i.e., which point to "acquire").

Algorithm 3 demonstrates how BO flow is executed to achieve the optimum. BO first builds a Gaussian process surrogate with a pre-generated initial dataset. Then the surrogate is updated iteratively with the sample created from acquisition function and its corresponding simulation value.

\subsection{Acquisition Function}

The most common acquisition functions applied in $\mathrm{BO}$ are probability of improvement (PI), expected improvement (EI), and lower confidence bound (LCB). In BO, the essence of PI and EI can be interpreted as an expected loss calculation based on the surrogate function value $f(x)$ at $x$. $x$ then is selected by minimizing the expected loss function. In this work, we will adopt these 


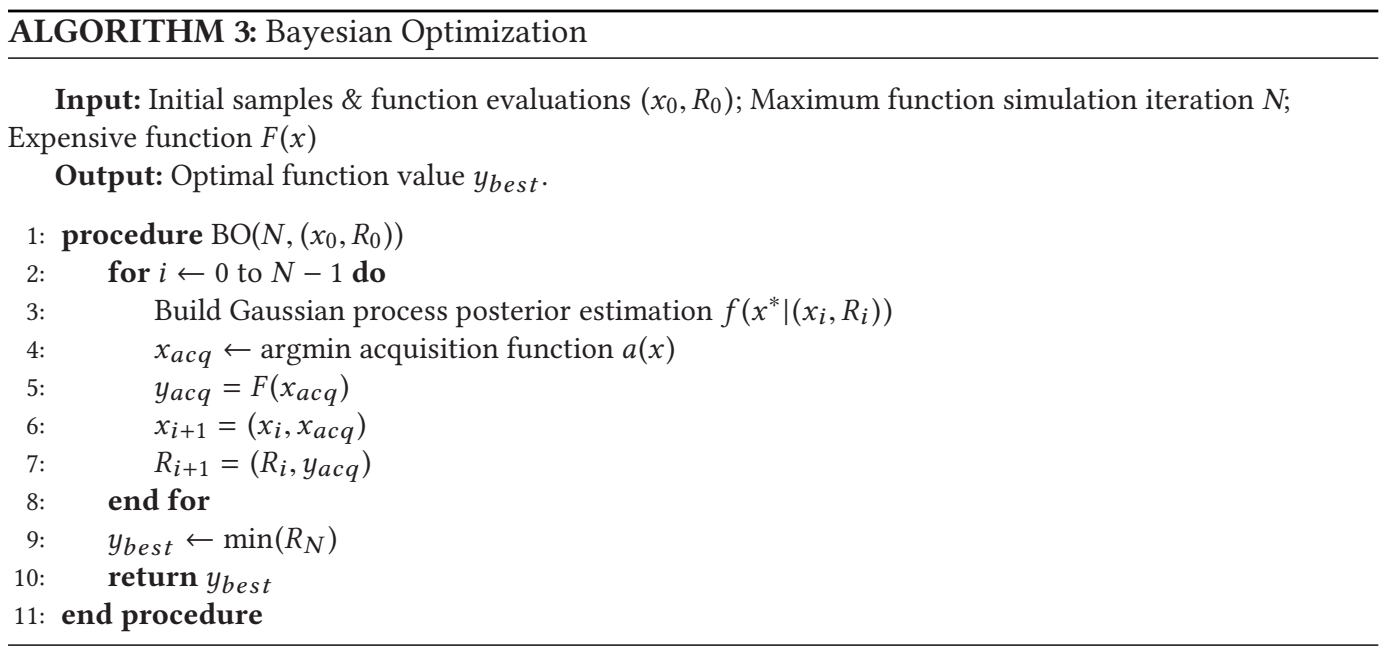

three methods to fulfill the EM simulation-driven optimization tasks and compare them with the proposed MFSMO-CS.

4.2.1 PI and El. The utility function of PI is

$$
u(x)=\left\{\begin{array}{ll}
0 & \mathrm{f}(\mathrm{x}) \geq f^{\prime} \\
1 & \mathrm{f}(\mathrm{x})<f^{\prime}
\end{array} .\right.
$$

PI would receive a unit reward when $f(x)$ is smaller than the current optimum $f^{\prime}$. Otherwise, PI rewards nothing. The PI acquisition function is expressed as

$$
a_{P I}=E[u(x) \mid x, D]=\Phi\left(f^{\prime} ; \mu(x), \sigma^{2}(x)\right) .
$$

The point with the largest PI value is selected as the next sample in the BO loop. Based on the description of PI's utility function, it actually does not take the precise improvement into consideration. Similarly, an alternative utility function called expected improvement (EI) that accounts for the decremental change between function value $f(x)$ and $f^{\prime}$ is developed as

$$
u(x)=\max \left(0,\left(f^{\prime}-f(x)\right) .\right.
$$

Then EI acquisition function is shown as

$$
a_{E I}=E[u(x) \mid x, D]=\left(f^{\prime}-\mu(x)\right) \Phi\left(f^{\prime} ; \mu(x), \sigma^{2}(x)\right)+\sigma^{2}(x) \phi\left(f^{\prime} ; \mu(x), \sigma^{2}(x)\right),
$$

where $\Phi$ and $\phi$ are the cumulative distribution function and probability density function of standard normal distribution.

4.2.2 $L C B$. The LCB acquisition function is shown as

$$
a_{L C B}=\mu(x)-\beta \sigma(x) .
$$

LCB does not follow the way of computing the expectation of utility function. However, all three acquisition functions described previously contain the model exploitation term $\mu(x)$ and design space exploration term $\sigma^{2}(x)$.

Consequently, each acquisition function provides a different degree of balance between exploitation and exploration. 


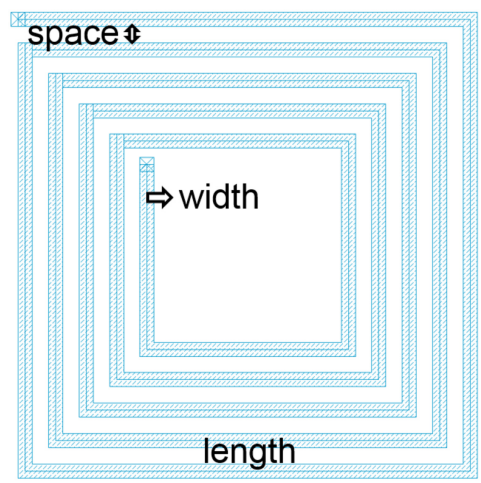

Fig. 4. Inductor layout.

Table 2. Inductor Design Summary

\begin{tabular}{|l|c|}
\hline Design Parameter & Design Range \\
\hline Length & 250 to 500 \\
\hline Width & 5 to 12 \\
\hline Space & 4 to 15 \\
\hline Turns (int) & 2 to 5 \\
\hline Performance & Desired Value \\
\hline$L$ & $2.5 \mathrm{nH}$ \\
\hline$Q$ & 12 \\
\hline
\end{tabular}

\section{EXPERIMENTAL RESULTS}

To demonstrate the effectiveness of the proposed MFSBO-CS methodology in an EM simulationbased optimization task, two design examples are explored. A comparison is drawn between the proposed method and the state-of-the-art $\mathrm{BO}$ with different acquisition function configurations. MFSBO-CS is implemented in Python and Matlab. The experiments were conducted with an Intel Xeon W3503 2.40-GHz CPU and 8 GB of memory.

\subsection{Inductor Design}

The layout of the square inductor is illustrated in Figure 4. It contains four tuning variables: space, width, length, and turn counts. This test case could be categorized as a mixed integer-continuous optimization problem since the turn counts of the inductor are discrete, whereas the rest are all continuous. Table 2 summarizes the inductor design. The low-fidelity simulation is implemented in ASITIC [18], whose simulation relies on the estimation of a simple pi model. We implement a subflow to generate ASITIC simulation configuration files and execute them in a batch mode. The high-fidelity simulation leverages a commercial 2.5D EM solver available at the university, and X11-GUITest [2] is applied for EM simulation automation. The S-parameter generated from EM solver is converted to an impedance matrix that can be used to calculate the inductance value and quality factor.

There are two inverse problems needed to be solved in the inductor case: (1) find the design variables' values for the inductance of $2.5 \mathrm{nH}$, and (2) find the design variables' values for the quality factor of 12 . Thus, the target functions are defined as $\|L-2.5 \mathrm{nH}\|$ and $\|Q-12\|$, respectively. 
Table 3. Optimization Results for Inductor Design

\begin{tabular}{cccccccl}
\hline Spec & Target & Method & Init. Sim. ConFigure & Opt. Iter. in HF & Opt. Solution & Opt. Value & Elapsed Time \\
\hline$L$ & $2.5 \mathrm{nH}$ & BO (PI) & $160 \mathrm{HF}$ & 45 & {$[500,5,4,5]$} & $4.428 \mathrm{nH}$ & $15,029.23 \mathrm{~s}$ \\
& & BO (EI) & $160 \mathrm{HF}$ & 9 & {$[500,5,4,5]$} & $4.428 \mathrm{nH}$ & $13,378.02 \mathrm{~s}$ \\
& & BO (LCB) & $160 \mathrm{HF}$ & 158 & {$[499.998,5,4,5]$} & $4.428 \mathrm{nH}$ & $16,256.46 \mathrm{~s}$ \\
& & MFSBO-CS & $100 \mathrm{LF}+60 \mathrm{HF}$ & 29 & {$[347.398,12,15,2]$} & $2.501 \mathrm{nH}$ & $5,001.54 \mathrm{~s}$ \\
\hline \multirow{2}{*}{$Q$} & 12 & BO (PI) & $160 \mathrm{HF}$ & 135 & {$[287.884,6.772,15,2]$} & 12.0001 & $14,420.16 \mathrm{~s}$ \\
& BO (EI) & $160 \mathrm{HF}$ & 160 & {$[398.695,12,15,2]$} & 12.0024 & $14,411.55 \mathrm{~s}$ \\
& BO (LCB) & $160 \mathrm{HF}$ & 151 & {$[400.179,12,15,2]$} & 12.0112 & $14,288.65 \mathrm{~s}$ \\
& MFSBO-CS & $100 \mathrm{LF}+60 \mathrm{HF}$ & 9 & {$[316.191,6.428,5.300,4]$} & 12.0992 & $5,442.17 \mathrm{~s}$ \\
\hline
\end{tabular}

HF, high-fidelity; LF, low-fidelity.

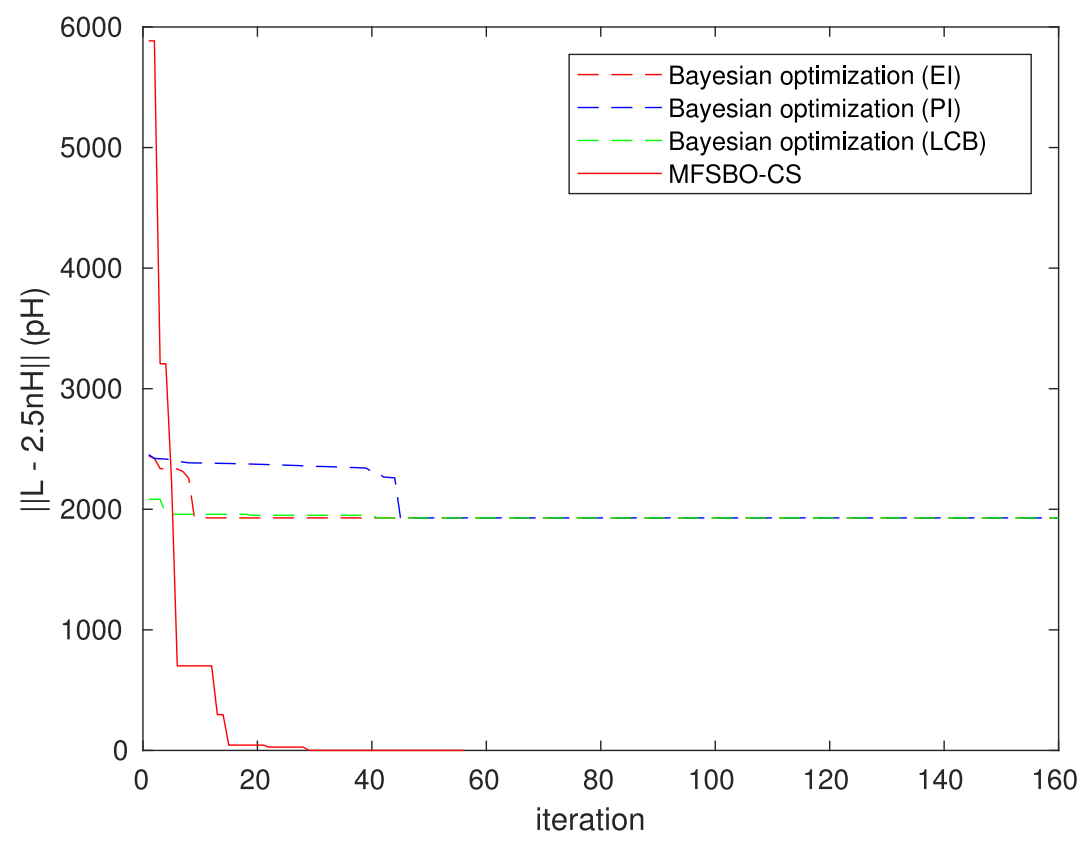

Fig. 5. Target function $\|L-2.5 \mathrm{nH}\|$ vs. number of high-fidelity samples.

5.1.1 Optimization Result Comparison for the Inductor. Table 3 summarizes the experimental results for the two specifications: inductance value and the quality factor at $2 \mathrm{GHz}$. Figures 5 and 6 demonstrate the optimization convergence plots for $L$ and $Q$ under different algorithms. In the baseline experiments, the budget of 160 EM simulations is set for each BO method. For the inductance problem, the result shows that optimal values are found at the 45th, 9th, and 158th iterations within 160 iterations for PI, EI, and LCB, respectively. All BO methods deliver a close final design given the simulation configuration shown in Table 3. The results indicate that the BO methods get stuck at the local optima [500,5,4,5].

A total budget of 100 low-fidelity simulations and 60 high-fidelity simulations is set for MFSBOCS. Due to the candidate point group 4 (G4) in the proposed adaptive sampling method and a surrogate with higher model accuracy, MFSBO-CS yields a much better and faster solution at the 29th EM simulation iteration out of 60 . The total elapsed simulation time is around one-third of BO. 


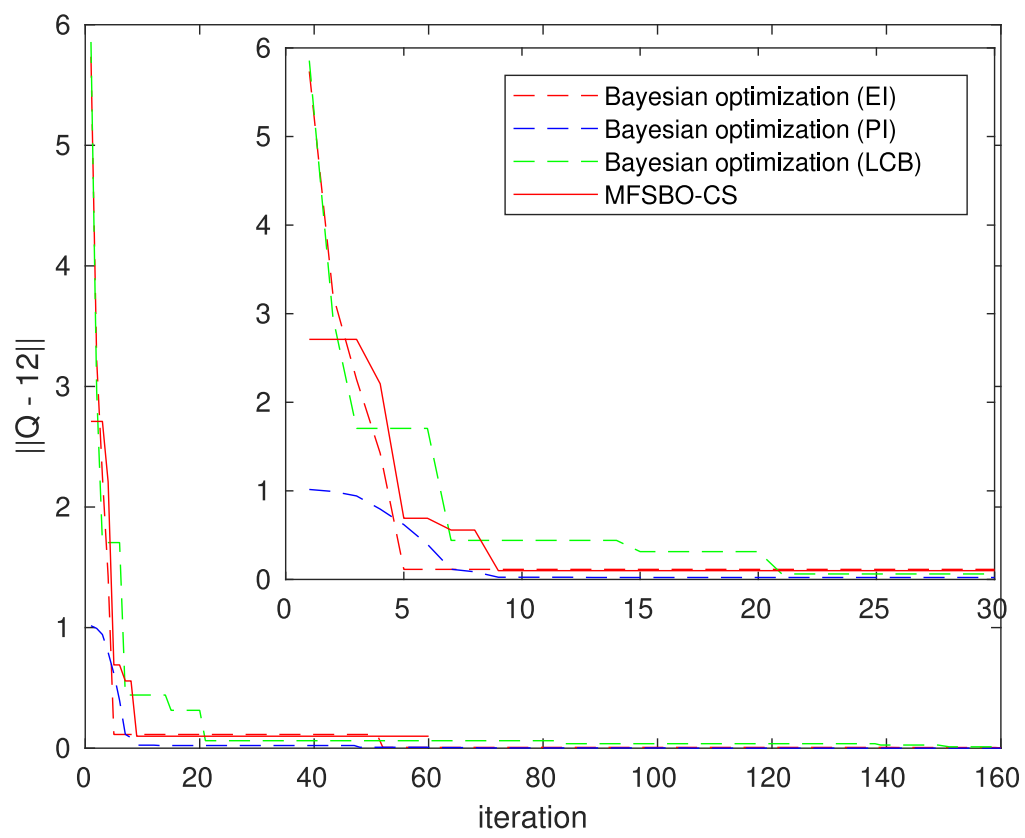

Fig. 6. Target function $\|Q-12\|$ vs. number of high-fidelity samples.

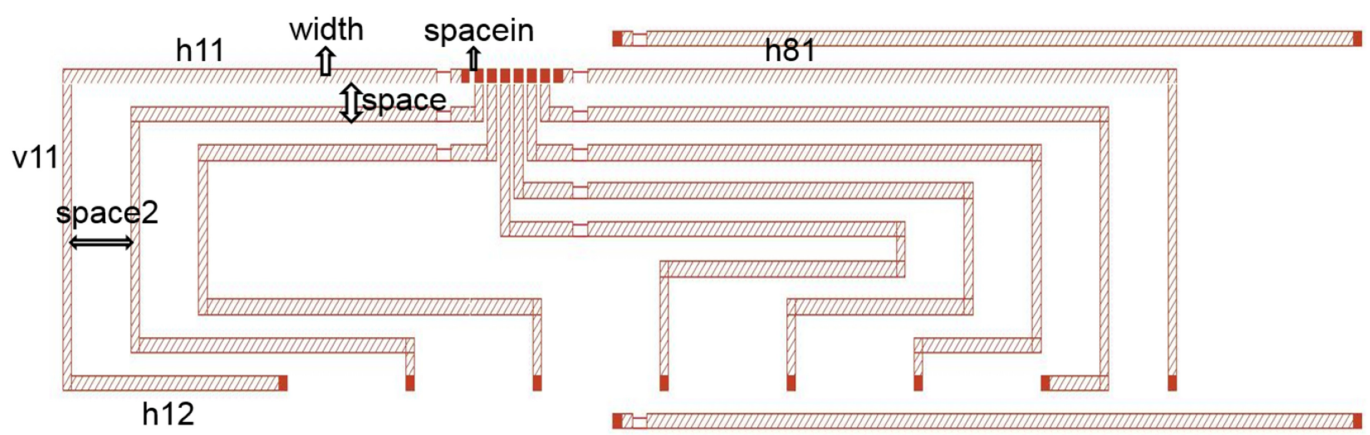

Fig. 7. Clock tree layout.

In the experiment of finding design variable values for a given quality factor, MFSBO-CS reaches a comparably final solution as BO methods, and it only takes 9 high-fidelity EM simulations after 100 low-fidelity simulations (approximately 17X faster than EI). The MFSBO-CS method finds a substantially more optimal solution than the $\mathrm{BO}$ methods. It did not get stuck in the local optimum like the BO methods did.

\subsection{Clock Tree Design}

The eight-channel clock tree example layout is illustrated in Figure 7 with six independent tuning design variables after considering some geometry constraints from the proposed structure. Two assumptions, approximately equal length of each metal channel and equal distance between all input ports and output ports, are applied to the layout design auto-generation. In the design shown in Figure 7, the slight wirelength difference between each channel is controlled by variable 
Table 4. Clock Tree Design Summary

\begin{tabular}{|l|c|}
\hline Design Parameter & Design Range \\
\hline spacein & 0.5 to 2 \\
\hline width & 0.5 to 1.5 \\
\hline delta1 & 0.6 to 2 \\
\hline h11 & 30 to 100 \\
\hline h12 & 30 to 80 \\
\hline delta2 & 0.6 to 2 \\
\hline Performance & Desired Range \\
\hline Maximum delay mismatching & $\leq 0.5$ ps \\
\hline Average delay & N/A \\
\hline
\end{tabular}

Table 5. Optimization Results for Clock Tree Design

\begin{tabular}{ccccccc}
\hline Opt. Obj. & Method & Init. Sim. ConFigure & Opt. Iter. in HF & Opt. Solution & Opt. Value & Elapsed Time \\
\hline Equation (28) & BO (PI) & $150 \mathrm{HF}$ & 77 & {$[0.5,0.5,2,30,67.678,2]$} & 1.121 & $18,093.53 \mathrm{~s}$ \\
& BO (EI) & $150 \mathrm{HF}$ & 101 & {$[0.5,0.5,2,65.193,40.810,0.6]$} & 1.135 & $18,410.80 \mathrm{~s}$ \\
& BO (LCB) & $150 \mathrm{HF}$ & 133 & {$[2,0.5,2,36.583,32.377,0.6]$} & 1.135 & $18,500.11 \mathrm{~s}$ \\
& MFSBO-CS & $100 \mathrm{LF}+50 \mathrm{HF}$ & 31 & {$[2,0.953,1.939,32.720,32.448,1.264]$} & 1.209 & $12,815.43 \mathrm{~s}$ \\
\hline
\end{tabular}

"spacein." Variable "width" is the width of the metal. Variable "space" is the vertical distance between two horizontal segments of adjacent channels. "h11" is defined as the length of the first horizontal segment in channel 1. Similarly, "h12" is the length of the second horizontal segment in channel 1. "v11" is the length of the first vertical segment in channel 1. "h81" and "space2" are eliminated by the geometry analysis of proposed layout. To avoid the violation of a design rule check (DRC) of the layout, variable "space" is defined as

$$
\text { space }=\text { width }+ \text { delta1 },
$$

where delta1 ranges from 0.6 to 2 . Also from the observation of the proposed layout shape, the critical length affecting the area of the layout would lie on the right side of the structure. To avoid a DRC issue from the two horizontal segments of channel 4, the total vertical length v11 is defined as

$$
v 11=7 * \text { space }+ \text { delta2 }
$$

where delta2 ranges from 0.6 to 2 . The design variables and specifications are summarized in Table 4.

The touchstone file generated from EM simulation is first translated to a time domain black box, then the black box is merged with the testbench circuit for SPICE simulation such that electrical characteristics could be extracted rather than just using the frequency domain information from EM simulation. For this test case, the flow is applied to optimize the multi-objective function containing two targets as follows: average_delay represents the average of total delays measured among eight channels, and delay_mismatching denotes the maximum skew of the eight channels. For simplicity, we formulate the multi-objective to be a simple weighted sum format as shown in Equation (28):

$$
y=\text { average_delay } / 1000+\text { delay_mismatching } .
$$




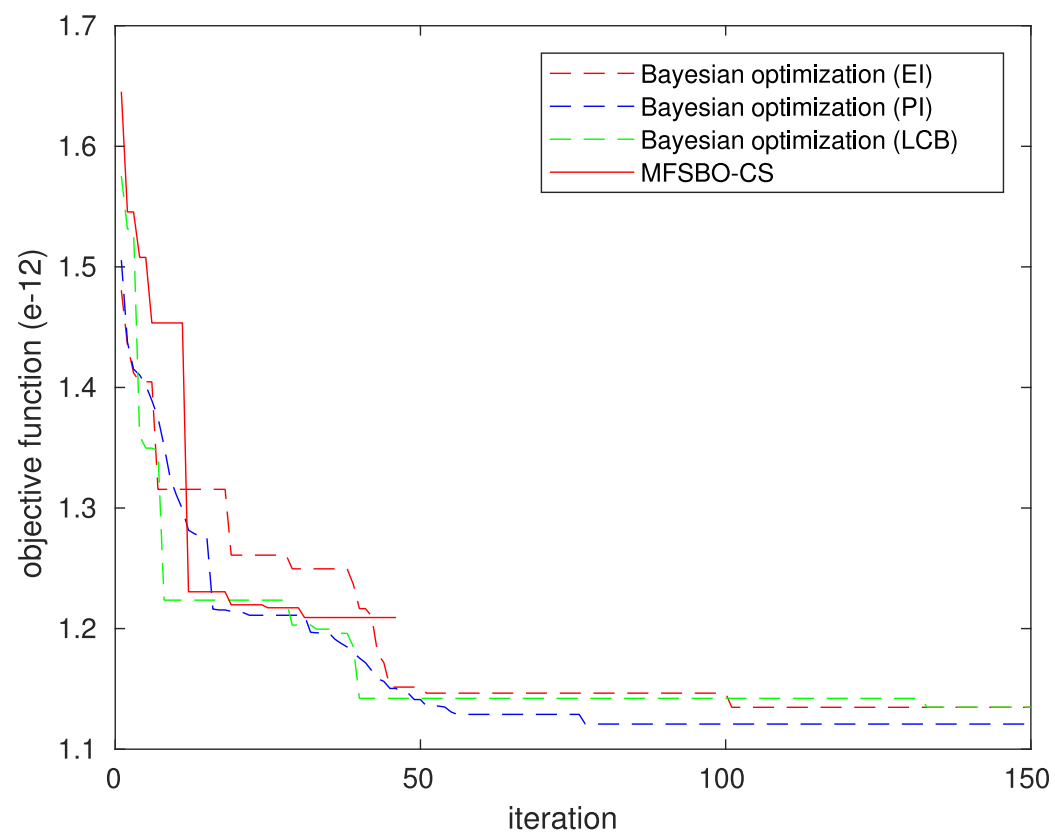

Fig. 8. Target function (28) vs. number of high-fidelity samples.

5.2.1 Optimization Results Comparison for Clock Tree Design. Table 5 summarizes the experimental results for the clock tree design. Figure 8 illustrates the optimization iterations with different algorithms. For the benchmark BO methods with different acquisition functions, a total of 150 EM simulations are executed and the optimal values are found at the 77th, 101th, and 133th iterations for PI, EI, and LCB, respectively. MFSBO-CS yields an optimal solution at the 31st EM simulation iteration out of 50 . Despite the approximately $5 \%$ improvement over MFSBO-CS, BO consumes almost $50 \%$ more elapsed time to complete. Through the observation of Figure 8 , the optimal solution from $\mathrm{BO}(\mathrm{LCB})$ at the 31 st iteration is 1.203 , which is almost same as the proposed MFSBO-CS.

\section{CONCLUSION}

With increasing importance of EM simulation in analog/RF system design, the computation cost of EM evaluation becomes a fundamental issue in analog/RF design automation. This article has presented a novel flow to accelerate EM simulation in analog/RF circuits design problems. This flow automates the process of the specific layout design generation and optimizes the layout based on the EM simulation results. In the optimization loop, we leverage the multi-fidelity EM simulation data along with the proposed parallel adaptive sampling method, sample filtering, and low-fidelity update mechanism to assist the system to quickly achieve the global optima at a low computation cost (i.e., a shorter simulation time). The experimental results show that the proposed method either reaches comparably or outperforms the optimization result from various BO methods with only approximately one- to two-thirds of the latter's computation elapsed time.

\section{REFERENCES}

[1] John W. Bandler, Radoslaw M. Biernacki, Shao Hua Chen, Piotr A. Grobelny, and Ronald H. Hemmers. 1994. Space mapping technique for electromagnetic optimization. IEEE Transactions on Microwave Theory and Techniques 42, 12 (1994), 2536-2544. 
[2] Dennis K. Paulsen. n.d. X11::GUITest. Retrieved June 11, 2020 from http://sourceforge.net/projects/x11guitest.

[3] Christodoulos A. Floudas. 1995. Nonlinear and Mixed-Integer Optimization: Fundamentals and Applications. Oxford University Press.

[4] Alexander I. J. Forrester, András Sóbester, and Andy J. Keane. 2007. Multi-fidelity optimization via surrogate modelling. Proceedings of the Royal Society A: Mathematical, Physical, and Engineering Sciences 463, 2088 (2007), 3251-3269.

[5] Karl Geiselhart, Lori Ozoroski, James Fenbert, Elwood Shields, and Wu Li. 2011. Integration of multifidelity multidisciplinary computer codes for design and analysis of supersonic aircraft. In Proceedings of the 49th AIAA Aerospace Sciences Meeting Including the New Horizons Forum and Aerospace Exposition. 465.

[6] Mehdi Ghoreyshi, Keneth Badcock, and Mark Woodgate. 2008. Integration of multi-fidelity methods for generating an aerodynamic model for flight simulation. In Proceedings of the 46th AIAA Aerospace Sciences Meeting and Exhibit. 197.

[7] Anthony Giunta, Robert Narducci, Susan Burgee, Bernard Grossman, William Mason, Layne Watson, and Raphael Haftka. 1995. Variable-complexity response surface aerodynamic design of an HSCT wing. In Proceedings of the 13th Applied Aerodynamics Conference. 1886.

[8] Geoffrey E. Hinton, Nitish Srivastava, Alex Krizhevsky, Ilya Sutskever, and Ruslan R. Salakhutdinov. 2012. Improving neural networks by preventing co-adaptation of feature detectors. arXiv:1207.0580.

[9] Marc C. Kennedy and Anthony O'Hagan. 2000. Predicting the output from a complex computer code when fast approximations are available. Biometrika 87, 1 (2000), 1-13.

[10] Slawomir Koziel and John W. Bandler. 2007. Microwave device modeling using space-mapping and radial basis functions. In Proceedings of the 2007 IEEE/MTT-S International Microwave Symposium. IEEE, Los Alamitos, CA, 799-802.

[11] Slawomir Koziel and John W. Bandler. 2007. A space-mapping approach to microwave device modeling exploiting fuzzy systems. IEEE Transactions on Microwave Theory and Techniques 55, 12 (2007), 2539-2547.

[12] Slawomir Koziel and John W. Bandler. 2012. Accurate modeling of microwave devices using kriging-corrected space mapping surrogates. International fournal of Numerical Modelling: Electronic Networks, Devices and Fields 25, 1 (2012), $1-14$.

[13] Slawomir Koziel, John W. Bandler, Ahmed S. Mohamed, and Kaj Madsen. 2005. Enhanced surrogate models for statistical design exploiting space mapping technology. In Proceedings of the 2005 IEEE MTT-S International Microwave Symposium Digest. IEEE, Los Alamitos, CA, 4.

[14] Cheng Li, Sunil Gupta, Santu Rana, Vu Nguyen, Svetha Venkatesh, and Alistair Shilton. 2018. High dimensional Bayesian optimization using dropout. arXiv:1802.05400.

[15] Wenlong Lyu, Pan Xue, Fan Yang, Changhao Yan, Zhiliang Hong, Xuan Zeng, and Dian Zhou. 2018. An efficient Bayesian optimization approach for automated optimization of analog circuits. IEEE Transactions on Circuits and Systems I: Regular Papers 65, 6 (2018), 1954-1967.

[16] Michael D. McKay, Richard J. Beckman, and William J. Conover. 2000. A comparison of three methods for selecting values of input variables in the analysis of output from a computer code. Technometrics 42, 1 (2000), 55-61.

[17] Juliane Müller, Christine A. Shoemaker, and Robert Piché. 2013. SO-MI: A surrogate model algorithm for computationally expensive nonlinear mixed-integer black-box global optimization problems. Computers \& Operations Research 40, 5 (2013), 1383-1400.

[18] Ali M. Niknejad. 2000. Analysis, Simulation, and Applications of Passive Devices on Conductive Substrates. Technical Report No. UCB/ERL M00/31. University of California, Berkeley.

[19] Martin Pelikan. 2005. Hierarchical Bayesian optimization algorithm In Hierarchical Bayesian Optimization Algorithm:Toward a New Generation of Evolutionary Algorithms. Studies in Fuzziness and Soft Computing, Vol. 170. Springer, 105-129.

[20] Ken Poulton, Robert Neff, Brian Setterberg, Bernd Wuppermann, Tom Kopley, Robert Jewett, Jorge Pernillo, Charles Tan, and Allen Montijo. 2003. A $20 \mathrm{GS} / \mathrm{s} 8 \mathrm{~b}$ ADC with a $1 \mathrm{MB}$ memory in $0.18 / \mathrm{spl} \mathrm{mu} / \mathrm{m}$ CMOS. In Proceedings of the 2003 IEEE International Solid-State Circuits Conference: Digest of Technical Papers (ISSCC'03). IEEE, Los Alamitos, CA, 318-496.

[21] Weiyi Qi. 2017. IC Design Analysis, Optimization and Reuse via Machine Learning. North Carolina State University.

[22] Peter Schvan, Jerome Bach, Chris Falt, Philip Flemke, Robert Gibbins, Yuriy M. Greshishchev, Naim Ben-Hamida, et al. 2008. A 24GS/s 6b ADC in 90nm CMOS. In Proceedings of the 2008 IEEE International Solid-State Circuits Conference: Digest of Technical Papers (ISSCC'08). IEEE, Los Alamitos, CA, 544-634.

[23] Shahriar Shahramian, Sorin P. Voinigescu, and Anthony Chan Carusone. 2009. A 35-GS/s, 4-bit flash ADC with active data and clock distribution trees. IEEE fournal of Solid-State Circuits 44, 6 (2009), 1709-1720.

[24] Jasper Snoek, Hugo Larochelle, and Ryan P. Adams. 2012. Practical Bayesian optimization of machine learning algorithms. In Advances in Neural Information Processing Systems. 2951-2959.

[25] Il'ya Meerovich Sobol'. 1967. On the distribution of points in a cube and the approximate evaluation of integrals. USSR Computational Mathematics and Mathematical Physics 7, 4 (1967), 784-802. 
[26] Michael Stein. 1987. Large sample properties of simulations using Latin hypercube sampling. Technometrics 29,2 (1987), 143-151.

[27] Guy A. E. Vandenbosch and Alexander Vasylchenko. 2011. A practical guide to 3D electromagnetic software tools. In Microstrip Antennas. InTech.

[28] R. A. Vural and T. Yildirim. 2012. Analog circuit sizing via swarm intelligence. AEU-International fournal of Electronics and Communications 66, 9 (2012), 732-740.

[29] David Warde-Farley, Ian J. Goodfellow, Aaron Courville, and Yoshua Bengio. 2013. An empirical analysis of dropout in piecewise linear networks. arXiv:1312.6197.

[30] Shuhan Zhang, Wenlong Lyu, Fan Yang, Changhao Yan, Dian Zhou, and Xuan Zeng. 2019. Bayesian optimization approach for analog circuit synthesis using neural network. In Proceedings of the 2019 Design, Automation, and Test in Europe Conference and Exhibition (DATE'19). IEEE, Los Alamitos, CA, 1463-1468.

Received June 2019; revised December 2019; accepted May 2020 